Agr. Biol. Chem., 39 (2), 541-544, 1975

\title{
The Binding Groups in Ovomucin-lysozyme Interaction
}

\author{
Akio Kato, Taiji Imoto and Kazuyoshi Yagishita \\ Department of Agricultural Chemistry, Faculty of Agriculture, \\ Yamaguchi University, Yamaguchi, Japan
}

Received October 3, 1974

\begin{abstract}
The ovomucin-lysozyme aggregation was remarkably affected by $\mathrm{pH}$ or ionic strength. The extent of interaction of F-ovomucin with lysozyme was much larger than that of S-ovomucin. The ovomucin-lysozyme interaction decreased correspondingly, at a rate depending on the time at which ovomucin was modified by neuraminidase. On the other hand, the ovomucin-lysozyme interaction disappeared completely by acetylation, succinilation, or carbamylation of lysyl $\varepsilon$-amino groups in lysozyme, but it was not greatly affected by guanidination of lysyl $\varepsilon$-amino groups in lysozyme. From these results, it was confirmed that the electrostatic interaction between the negative charges of the terminal sialic acid in ovomucin and the positive charges of lysyl $\varepsilon$-amino groups in lysozyme is essential for the ovomucin-lysozyme interaction.
\end{abstract}

It is known that ovomucin, the structural glycoprotein of egg white, interacts with lysozyme. $^{(\sim 4)}$ The ovomucin-lysozyme interaction may play an important part in formation of the rigid gelatinous structure in the thick white. Cotterill and Winter suggested that the ovomucin-lysozyme interaction are mainly electrostatic. ${ }^{11} \quad$ Robinson and Monsey suggested that the electrostatic interaction between the negative charges of ovomucin molecules and the positive charges of lysozyme molecules is important for the interaction. ${ }^{4}$ However, there is no direct evidence for the suggestion.

This paper describes the binding groups in ovomucin-lysozyme interaction.

\section{MATERIALS AND METHODS}

Materials. A lysozyme preparation isolated from hen's egg white by the direct crystallization method was recrystallized five times and lyophilized. Trypsin was a product from Worthington Biochemical Co. and pronase was from Kaken Chemical Co. Neuraminidase from $\mathrm{Cl}$. perfringens was a product of Sigma Chemical Co. All other reagents employed were analytical grade.

Preparation of ovomucin. The procedure employed to prepare ovomucin has been described previously. ${ }^{51}$ The fresh thick white was separated into gel and sol parts by ultracentrifugation for $60 \mathrm{~min}$ at $59,000 \times g$. The gel part was washed directly with $2 \% \mathrm{KCl}$ solu- tion without diluting with water until the washing was free from proteins. The gel-like precipitate was finally washed with water until the washing was free from chloride. Ovomucin thus obtained was solubilized by $0.01 \mathrm{~m}$ mercaptoethanol in carbonate buffer $(\mathrm{pH}$ 9.6, ionic strength 0.25 ). After solubilization, ovomucin was dialyzed against one hundred volumes of $1 / 15 \mathrm{M}$ phosphate buffer ( $\mathrm{pH}$ 5.4). The reduced ovomucin solution thus obtained was employed in the following experiments.

Separation of ovomucin into two components. Details of the procedure employed to separate reduced ovomucin into two components have been described in the previous paper.61 The separation was carried out by preparative electrophoresis using density gradient column. The fast moving component is named as F-ovomucin and the slow moving component as Sovomucin. ${ }^{6 !}$

Action of enzymes on ovomucin. To $0.5 \%$ solution of reduced ovomucin were added $0.01 \%$ proteolytic enzymes and the mixtures were incubated at $30^{\circ} \mathrm{C}$ for $4 \mathrm{hr}$ in $0.05 \mathrm{M}$ Tris- $\mathrm{HCl}$ buffer ( $\mathrm{pH} \mathrm{8.0)}$ ).

$5 \mu \mathrm{g}$ of neuraminidase ( 1.5 units $/ \mathrm{mg}$ ) was added to $10 \mathrm{ml}$ of $0.2 \%$ solution of reduced ovomucin. Enzymatic action was carried out at $30^{\circ} \mathrm{C}$ for $24 \mathrm{hr}$ in $1 / 15 \mathrm{M}$ phosphate buffer ( $\mathrm{pH} 5.4$ ).

Modification of lysozyme. Acetylation of lysozyme was carried out according to the method described by Yamasaki et al..$^{71}$ with a slight modification. To a mixture of $10 \mathrm{ml}$ of $5 \%$ aqueous lysozyme solution and $10 \mathrm{ml}$ of saturated sodium acetate solution was added dropwise $5 \mathrm{~g}$ of acetic anhydride over a period 
of $1 \mathrm{hr}$ at $0^{\circ} \mathrm{C}$.

Guanidination, carbamylation, and succinilation of lysozyme were carried out according to the methods described by Kassell et al., ${ }^{81}$ Stark et al.,91 and Chu et al., ${ }^{101}$ respectively.

These modifications of lysozyme are specific for the amino groups in lysozyme. The extent of each modifications was determined spectrophotometrically using a specific reagent for amino groups, trinitrobenzenesulfonate, according to the method described by Haynes et al. ${ }^{11}$

Measurement of the extent of ovomucin-lysozyme interaction. $\quad 0.2 \mathrm{ml}$ of $0.2 \%$ reduced ovomucin solution was added to $2 \mathrm{ml}$ of $0.1 \%$ lysozyme solution in $1 / 15 \mathrm{M}$ phosphate buffer containing $0.1 \%$ gelatin. The turbidity of reduced ovomucin-lysozyme aggregation was stabilized by addition of $0.1 \%$ gelatin. The mixture was shaked and allowed to stand at $20^{\circ} \mathrm{C}$. After $20 \mathrm{~min}$, turbidity was determined by measuring absorbances at $550 \mathrm{~nm}$. The extent of ovomucinlysozyme interaction was shown as percentages of the turbidity of reduced ovomucin-lysozyme mixture in $1 / 15 \mathrm{M}$ phosphate buffer ( $\mathrm{pH} 5.4$ ).

\section{RESULTS}

The ovomucin-lysozyme aggregation was remarkably affected by $\mathrm{pH}$ or ionic strength,

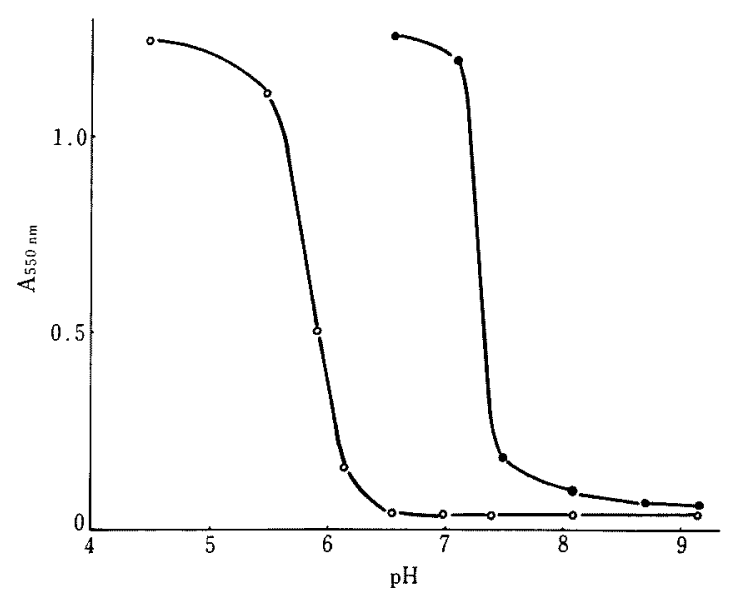

FIG. 1. Effect of $\mathrm{pH}$ on Ovomucin-lysozyme Aggregation.

Aggregation was measured in $1 / 15 \mathrm{M}$ phosphate buffer $(0-0)$ and in $1 / 30 \mathrm{M}$ phosphate buffer $(-\bullet)$.

as shown in Figs. 1 or 2. The transition $\mathrm{pH}$ of ovomucin-lysozyme aggregation was also affected by ionic strength, as shown in Fig. 1. There were no other effects of addition of gela-

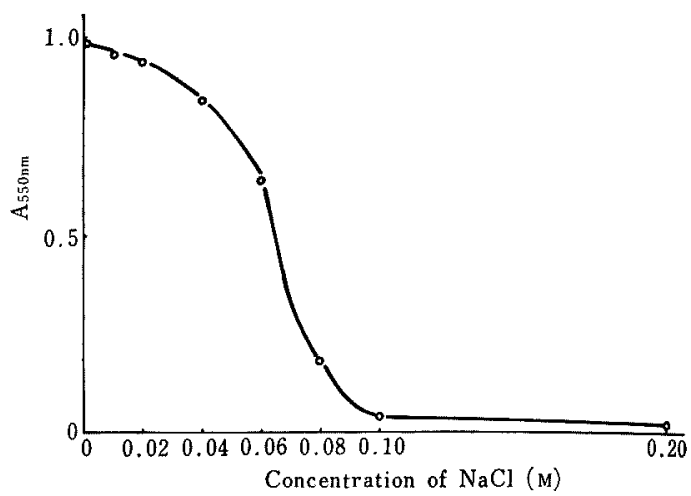

FIG. 2. Effect of Ionic Strength on Ovomucinlysozyme Aggregation.

Aggregation was measured in $1 / 15 \mathrm{M}$ phosphate buffer ( $\mathrm{pH}$ 5.4) and then $\mathrm{NaCl}$ was added.

tin on ovomucin-lysozyme aggregation than the stabilization of turbidity.

The extent of interaction of $\mathrm{F}$-ovomucin with lysozyme was much larger than that of $S$ ovomucin, as shown in Table I. The extent of

Table I. EfFect of Modification of Ovomucin ON OVOMUCIN-LYSOZYME INTERACTION

\begin{tabular}{lc}
\hline \multicolumn{1}{c}{ Ovomucin } & The extent of interaction \\
\hline Control & 100 \\
F-Ovomucin & 166 \\
S-Ovomucin & 16 \\
Trypsin-digested & 169 \\
Pronase-digested & 154 \\
Neuraminidase-digested & 3 \\
\hline
\end{tabular}

ovomucin-lysozyme interaction increased by the digestion of ovomucin with proteolytic enzyme, as shown in Table I.

The extent of ovomucin-lysozyme interaction decreased correspondingly, at a rate depending on the time at which ovomucin was modified by neuraminidase, as shown in Fig. 3 .

The extent of modification of the amino groups in lysozyme was 3.4 in guanidination, 6.0 in acetylation, 7.0 in succinilation, and 5.5 in carbamylation, as shown in Table II. By these modifications except guanidination, the ovomucin-lysozyme interaction in $1 / 15 \mathrm{~m}$ phosphate buffer (pH 5.4) disappeared completely, but it remained $80 \%$ by guanidination. 


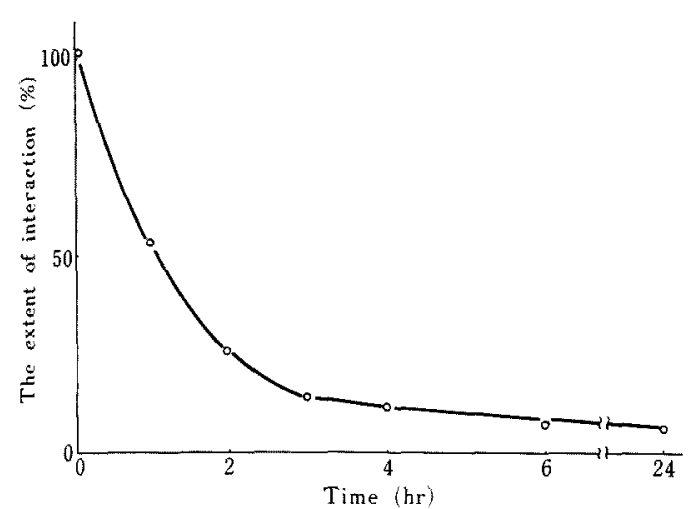

Fig. 3. Effect of Neuraminidase Action for Ovomucin on Ovomucin-lysozyme Interaction.

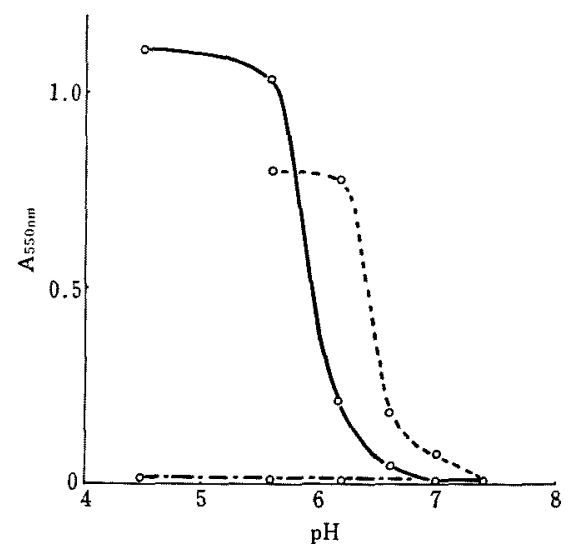

Fig. 4. Effect of pH on Ovomucin-modified lysozyme Aggregation.

$(\mathrm{O}-\mathrm{O})$, ovomucin-lysozyme aggregation; $(\mathrm{O}-\mathrm{O})$, ovomucin-guanidinated lysozyme aggregation; $(\mathrm{O}-\mathrm{-}-\mathrm{O})$, ovomucin-acetylated lysozyme aggregation.

The ovomucin-guanidinated lysozyme aggregation was affected by $\mathrm{pH}$, as well as ovomucinlysozyme aggregation, and the transition $\mathrm{pH}$ of its aggregation was higher than that of ovomucin-lysozyme aggregation, as shown in Fig. 4. The ovomucin-acetylated lysozyme aggregation did not occur in any $\mathrm{pH}$.

Dependence of lysozyme concentration on ovomucin-lysozyme aggregation in the presence of $0.4 \mathrm{mg}$ reduced ovomucin was shown in Fig. 5. Curve of ovomucin-lysozyme aggregation indicated to increase in proportion to lysozyme concentration up to $0.75 \mathrm{mg}$ lysozyme but to get at saturation over about $1.0 \mathrm{mg}$ lysozyme.

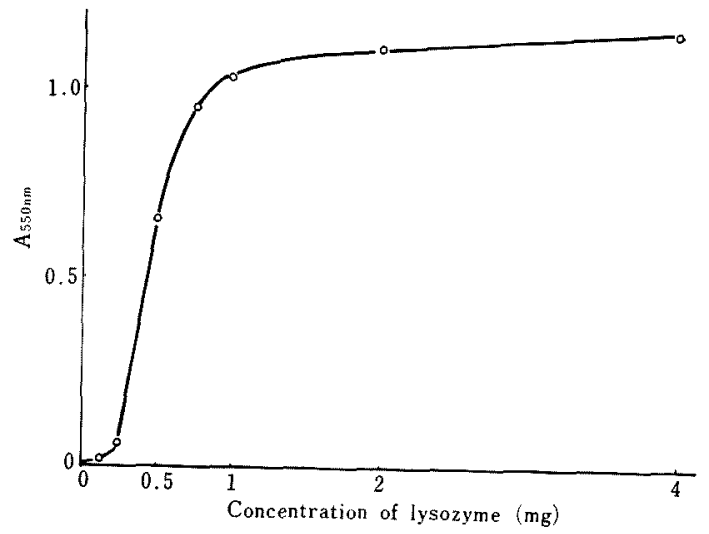

FIG. 5. Dependence of Lysozyme Concentration on Ovomucin-lysozyme Aggregation.

Aggregation was measured in the presence of $0.4 \mathrm{mg}$ reduced ovomucin.

\section{DISCUSSION}

The ovomucin-lysozyme interaction seems to be electrostatic estimating from the effects of $\mathrm{pH}$ or ionic strength on its interaction, as proposed by some workers. ${ }^{1,4)}$

It was shown in the previous report that $75 \%$ of the terminal sialic acid were released by neuraminidase action on ovomucin. ${ }^{12}$ The ovomucin-lysozyme interaction decreased correspondingly, at a rate depending on the enzymatic removal of sialic acid from ovomucin. Though the contaminated proteolytic enzymes in neuraminidase were expected, the effects of these enzymes on ovomucin-lysozyme interaction are not possible because the interaction did not decrease but increased reversely by the action of proteolytic enzymes on ovomucin. The increase of the interaction by the action of proteolytic enzymes on ovomucin may reflect the fact that the binding groups of ovomucin were exposed more outside. In fact, it was shown in the previous report that ovomucin is relatively resistant to these enzymes and a part of protein portions is digested but the carbohydrate protions remain to be macromolecules. ${ }^{13)}$ In addition to these results, the interaction of F-ovomucin, sialic acid rich component, with lysozyme was much stronger than that of S-ovomucin, sialic acid poor component. These results suggest that the terminal 
Table II. EFFECT OF Modification of Lysozyme ON OVOMUCIN-LYSOZYME INTERACTION

\begin{tabular}{lcc}
\hline \multicolumn{1}{c}{ Lysozyme } & $\begin{array}{c}\text { Extent of } \\
\text { modification }\end{array}$ & $\begin{array}{c}\text { Extent of } \\
\text { interaction }\end{array}$ \\
\hline Control & 0 & 100 \\
Guanidinated & 3.4 & 80 \\
Acetylated & 6.0 & 0 \\
Succinilated & 7.0 & 0 \\
Carbamylated & 5.5 & 0 \\
\hline
\end{tabular}

sialic acid of ovomucin is essential or the ovomucin-lysozyme interaction.

On the other hand, the ovomucin-lysozyme interaction disappeared completely by acetylation, succinilation, or carbamylation of amino groups in lysozyme. There are six lysyl $\varepsilon$ amino groups and an $\alpha$-amino group in lysozyme. The positive charges of lysyl $\varepsilon$-amino groups in lysozyme were diminished below two by these modification. The main feature of ovomucin-lysozyme interaction was not affected by guanidination of lysozyme, but the transition $\mathrm{pH}$ of its aggregation was higher and total turbidity was lower than that of native lysozyme. Only lysyl $\varepsilon$-amino groups are modified by guanidination, as shown in the previous report. ${ }^{8)}$ This may reflect the fact that the positive charges of lysyl $\varepsilon$-amino groups in lysozyme converted to stronger base by this modification. These results suggest that the positive charges of lysyl $\varepsilon$-amino groups in lysozyme is essential for ovomucin-lysozyme interaction.

Therefore, it was confirmed that the electrostatic interaction between the negative charges of the terminal sialic acid in ovomucin and the positive charges of lysyl $\varepsilon$-amino groups in lysozyme is essential for the ovomucin-lysozyme interaction.
Calculating from dependence of lysozyme concentration on ovomucin-lysozyme interaction, it leads to the assumption that the ovomucin-lycozyme interaction may consist of one molar lysozyme and two molar sialic acid in ovomucin, that is, two lysyl $\varepsilon$-amino groups in one molar lysozyme may interact with two sialic acid in ovomucin.

It will be interesting to study whether sialoglycoprotein such as ovomucin interacts specifically with lysozyme or generally with some basic proteins.

Acknowledgement. The authors thank Mr. S. Sumi for excellent technical assistance.

\section{REFERENCES}

1) O. J. Cotterill and A. R. Winter, Poultry Sci, 34, 679 (1955).

2) J. Brooks and H.P. Hale, Biochim. Biophys. Acta, 46, 289 (1961).

3) A. Kato, R. Nakamura and Y. Sato, Agr. Biol. Chem., 35, 351 (1971).

4) D. S. Robinson and J. B. Monsey, J. Sci. Fd. Agric., 23, 893 (1972).

5) A. Kato, R. Nakamura and Y. Sato, Agr. Biol. Chem., 34, 1009 (1970).

6) A. Kato and Y. Sato, ibid., 36, 831 (1972).

7) N. Yamasaki, K. Hayashi and M. Funatsu, ibid., 32, 55 (1968).

8) B. Kassell and R. B. Chow, Biochemistry, 5, 3449 (1966).

9) G. R. Stark, W. H. Stein and S. Moore, J. Biol. Chem., 235, 3177 (1960).

10) F.S. Chu, E. Crary and M.S. Bergdoll, Biochemistry, 8, 2890 (1969).

11) R. Haynes, D. T. Osuga and R. E. Feeney, ibid., 6, 541 (1967).

12) A. Kato, H. Hayashi and K. Yagishita, Agr. Biol. Chem., 38, 1137 (1974).

13) A. Kato, K. Fujinaga and K. Yagishita, ibid., 37, 2479 (1973) 